http://jmscr.igmpublication.org/home/

ISSN (e)-2347-176x ISSN (p) 2455-0450

crossref DOI: https://dx.doi.org/10.18535/jmscr/v8i4.03

Journal Of Medical Science And Clinical Research

IGM Publication

An Official Publication of IGM Publication

\title{
Role of CT in the Evaluation of Buccal Mucosa Malignancy
}

\author{
Authors \\ Dr Sapna Patel ${ }^{1}$, Dr Sanjiv Patel ${ }^{2}$, Dr Darshan Thummar ${ }^{3}$ \\ ${ }^{1}$ Assi Prof, Dept of Radiology, GCRI \\ ${ }^{2}$ Assi Prof, Dept of Radiology, GCRI \\ ${ }^{3}$ Resident Doctor, GCRI
}

\section{Introduction}

The most common oral cavity cancer in India is buccal mucosal carcinoma. The high incidence of buccal mucosa carcinoma in our country is due to the widespread use of tobacco in different forms, and the locally advanced cancers account for about 70 percent of the cases at presentation ${ }^{[1-5]}$.

Although the cancer can be visualized immediately on clinical evaluation, it is difficult to assess its deeper extension

With the advent of Computed Tomography (CT), the staging of buccal mucosa tumours has become more accurate, leading to proper treatment planning and execution.

The advent of computed tomography (CT) has made the staging of buccal mucosal tumors more precise, leading to proper treatment planning and execution.

There are several international consensus recommendations for the treatment of oral cavity cancers, but none of them specifically address buccal mucosal cancers.

Clearly, there is an urgent need to formulate consensus statements for the management of buccal mucosa carcinoma based on Indian data and experience, which would not only incorporate the available evidence but would also be practicable in Indian hospitals.

\section{Aims and Objectives}

- To evaluate the role of CT scan in diagnosis of buccal mucosal malignancies and its characteristics.

- Role of CT scan in staging of buccal mucosal malignancies to determine surgical resectibility and their prognosis.

- To evaluate recurrent or residual lesions in treated patients.

- To guide surgeon/clinical oncologist in treatment planning \& to determine prognosis.

\section{Materials and Methods}

During the period from June 2018 to November 2019 prospective study of 50 patients of various age was carried out.

\section{Inclusion Criteria}

- All patients diagnosed and suspicious of lesion involving buccal space.

- Patients who have already received some treatment in the form of surgery or chemotherapy or radiotherapy.

\section{Exclusion Criteria}

- Patients having allergy from contrast material used in CT.

- Patients presenting to radiology department having buccal mucosal 
malignancy in past and are cured completely will be excluded from the study.

- Patients not willing to participate in the study.

\section{CT Scan Checklist For CA Buccal Mucosa}

- Epicenter and dimensions of the lesion.

- Soft tissue extent (lateral, superior and medial) overlying skin, maxillary sinus, paramandibular extent, lingual muscles, BOT and FOM (T4a).

- Soft tissue extentposteriorly to RMT, pterygomaxillary fissure, pterygopalatine fossa, pterygoid plates and masticator space/ITF (T4b) with supra or infra mandibular notch extent.

- Bone erosion and extent mandible (height and AP extent) and maxilla.

- Nodal status - number \& size of abnormal nodes, level, presence of necrosis/ extracapsular spread, invasion of adjacent structures and vessels.

\section{Observations and Discussion}

In study

The age of patients ranges from 31 to 70 years. These were 39 males and 11 female patients yielding a male to female ratio of 3.55:1.0.

38 out of 50 patients have primary lesion of $>4$ $\mathrm{cm}$ upgrading its stage to $\mathrm{T} 3$ and more.

31 out of 50 patients have depth of invasion into adjacent space of $>6 \mathrm{~mm}$ greatly affecting management option as tumor thickness of more than $5 \mathrm{~mm}$ was found to be associated with treatment failure and lymph node metastases.

In study, 21 out of 50 patients showed T4 stage at times of presentation followed by 17 patients with T3 disease. Only 9 out of 50 patients showed T2 stage followed by 3 out of 50 patient showed stage $\mathrm{T} 1$ disease.

In study, most common nodal staging group was $\mathrm{N} 2(54 \%)$ followed by N0 (28\%), N1 (12\%) and N3 (6\%).
Only 2 out of 50 patient showed distant metastasis involving lungs and brain with rest of patients do not show any features of distant metastasis.

\section{Histopathological Grading}

All 50 out of 50 patients were proved to be squamous cell carcinoma out of which most common histopathological grading was well differentiated tumours $(48 \%)$ followed by moderately differentiated (34\%) and poorly differentiated tumours (18\%).

Final stage on HPE

\begin{tabular}{|l|c|c|}
\hline Final stage & No. of Patients & Percentage (\%) \\
\hline I & 3 & $6 \%$ \\
\hline II & 8 & $16 \%$ \\
\hline III & 6 & $12 \%$ \\
\hline IV & 33 & $66 \%$ \\
\hline
\end{tabular}

Treatment strategies according to tumour final stage only $8(20 \%)$ of them underwent postoperative radiotherapy or chemotherapy as rest of cases were not fit for surgery. Overall, $11(22 \%)$ patients were treated with surgery followed by radiotherapy or chemotherapy and rest of cases were treated by radiotherapy alone 4 (8\%), chemotherapy alone 17 (34\%) and combined radiotherapy and chemotherapy $18(36 \%)$.

Out of 11 patients that underwent surgery, 3 (27.28\%) patients developed recurrent lesion after surgery.

\section{Stage T3N1}

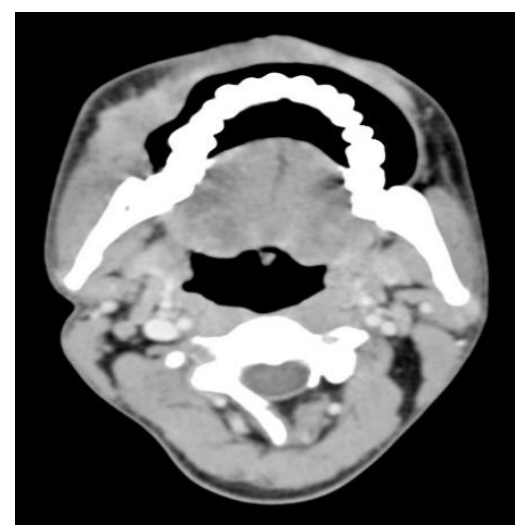



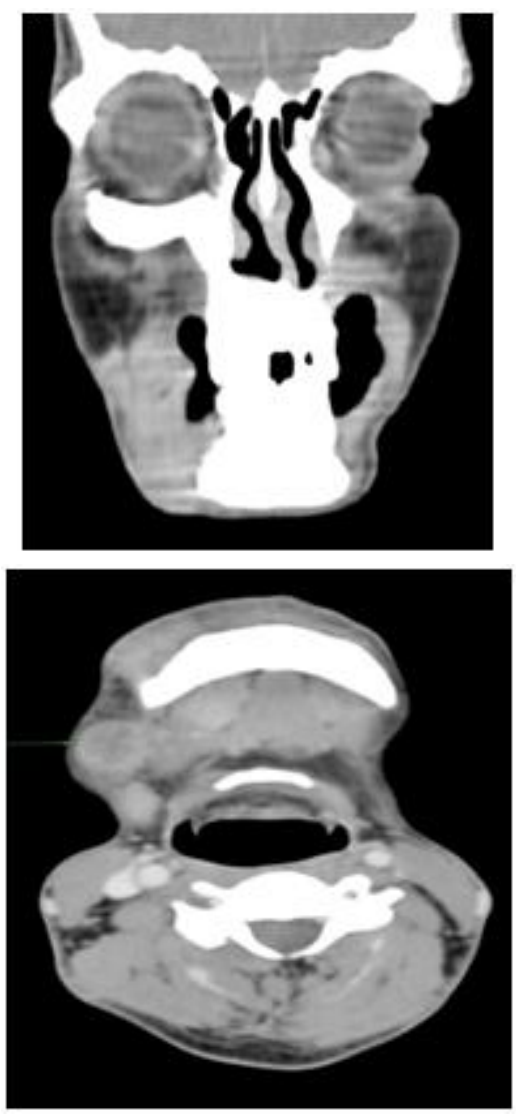

Case of T3N1 tumour: Axial and coronal CECT images showing heterogeneously enhancing soft tissue density lesion on right side of buccal space $>4 \mathrm{~cm}$ in greatest dimension with single enlarged necrotic node in right level IB.

\section{Stage T4M1}
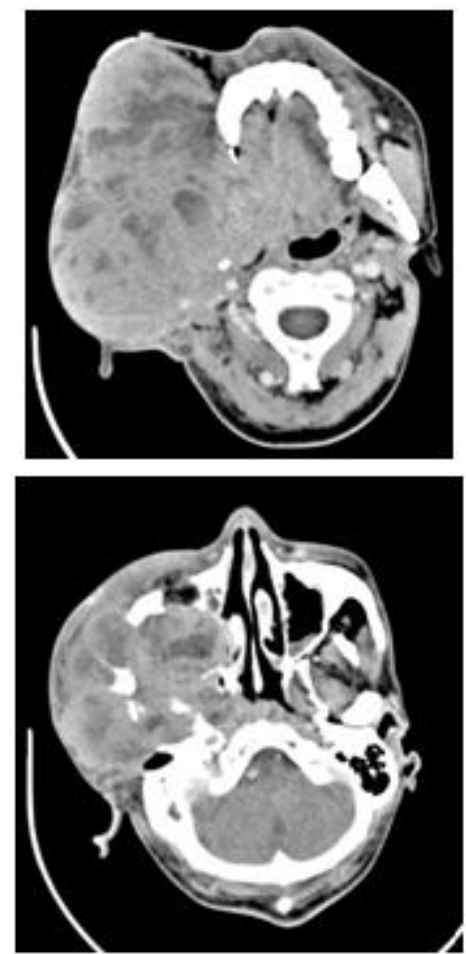
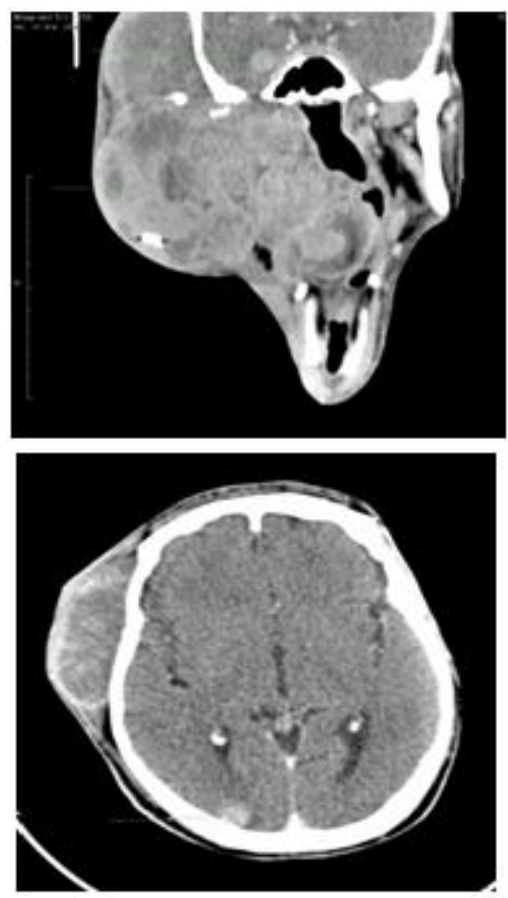

Case of T4M1 tumour: Axial and coronal CECT images showing large heterogeneously enhancing soft tissue density lesion on right side of buccal space $>4 \mathrm{~cm}$ in greatest dimension extending into right masticator space, right infra temporal and temporal fossa, reaching upto base of skull, invading right maxillary sinus encasing right CCA and ICA with two heterogeneously enhancing lesions involving right temporal and right occipital region s/o brain metastasis.

\section{Recurrence}
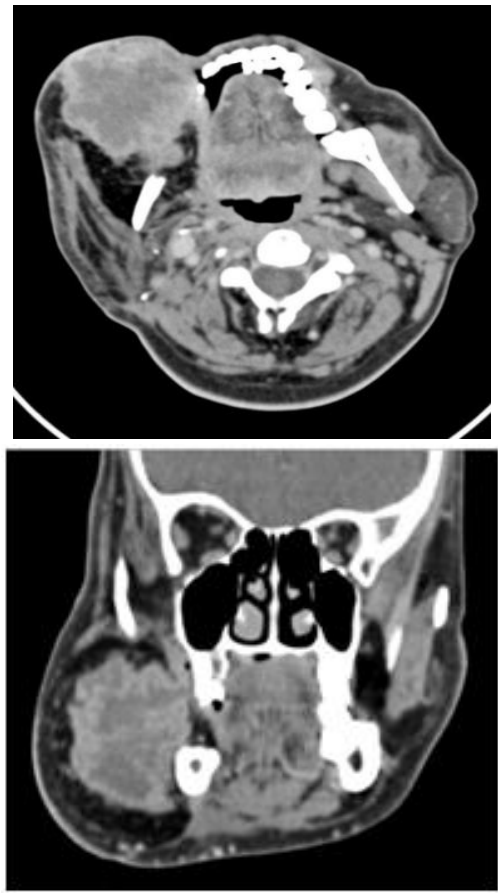
Operated case of right buccal space malignancy: Axial and coronal CECT images showing large heterogeneously enhancing soft tissue density lesion at operated site involving reconstructed myo-cutaneous flap.

\section{Conclusion}

Purpose for imaging the buccal mucosal malignancy is primarily to determine the origin and extent of the lesion, locoregional and distal metastasis with knowledge of the expected CT imaging findings can be helpful for the radiologist to diagnose lesion and its staging.

CT is also helpful modality to suggest recurrence in operated cases of buccal mucosal malignancy.

The study reports advanced stage of diagnosis and most of cases had well differentiated tumor. Hence, it is anticipated that early detection can reduce morbidity of buccal mucosa carcinoma.

The study warrants implementing social awareness about early sign and symptoms education on self oral screening methods so as to avoid the risk of late presentation of oral squamous cell carcinoma.

\section{Bibliography}

1. National Cancer Registry Programme, ICMR: Three year report of PBCRs 20062008.

2. National Cancer Registry Programme, ICMR: Consolidated report of Hospital Based Registries 2004-2006.

3. Parkin DM, Bray F, Ferlay J, Pisani P. Estimating the world cancer burden. Int $\mathbf{J}$ Cancer 2001 Oct 15; 94(2):153- 6.

4. Pradhan SA. Surgery for the cancer of the Buccal Mucosa. SeminSurgOncol. 1989; 5(5): 318-21.

5. Iyer SG, Pradhan SA, Pai PS, Patil S. Surgical treatment outcomes of localized squamous carcinoma of Buccal Mucosa. Head Neck 2004 Oct; 26(10): 897-902. 